\title{
Quench Analysis of a High-Current Forced-Flow HTS Conductor Model for Fusion Magnets
}

\author{
Claudio Marinucci, Luca Bottura, Marco Calvi, and Rainer Wesche
}

\begin{abstract}
A fundamental understanding of the quench phenomenon is particularly important in the design and operation of magnets using High Temperature Superconductors because the quench propagation velocity is low due to high specific heat at high temperature. We have performed the simulations of a high-current forced-flow conductor model to assess the key quench parameters, e.g. the temperature increase of the normal zone and its propagation velocity, using the CryoSoft code THEA. The sensitivity of these results on conductor design and operation parameters was assessed with a variational analysis. Results show that the helium flow has a beneficial impact on the longitudinal propagation of the normal zone and the resulting reduction of the peak quench temperature.
\end{abstract}

Index Terms-HTS, normal zone propagation, numerical simulation, quench, thermohydraulic analysis.

\section{INTRODUCTION}

$\mathbf{H}$ IGH Temperature Superconductors (HTS) are considered a serious alternative to Low Temperature Superconductors (LTS) for magnets of future fusion devices because of several potential advantages, e.g. high operation temperature, high temperature margin, high magnetic field properties, cryogenic power saving, etc. For these applications (RE)BCO (Rare-Earth Barium Copper Oxide) coated conductors are preferred to other HTS conductors because of their high critical current density in high magnetic fields and the potential of lower cost. Studies and experiments of quench propagation in HTS tapes have shown that because of the high temperature margin and the rapidly increasing specific heat with increasing temperatures, HTS coils can have low propagation velocity of the normal zone [1] making the quench detection and protection a difficult task [2].

We have studied the quench behavior of a full size high current (RE)BCO conductor, i.e. a stack of tapes and a cooling channel, using the CryoSoft code THEA (Thermal, Hydraulic and Electric Analysis of Superconductor Cables) [3]. The analysis is performed in a broad range of model and physical parameters.

Manuscript received August 02, 2010; accepted September 21, 2010. Date of publication November 22, 2010; date of current version May 27, 2011.

C. Marinucci and R. Wesche are with EPFL-CRPP, Fusion Technology, 5232 Villigen-PSI, Switzerland (e-mail: claudio.marinucci@ psi.ch).

L. Bottura is with CERN, AT-MSC, 1211 Geneva, Switzerland.

M. Calvi is with PSI, 5232 Villigen-PSI, Switzerland.

Color versions of one or more of the figures in this paper are available online at http://ieeexplore.ieee.org.

Digital Object Identifier 10.1109/TASC.2010.2090329

\section{HTS CONDUCTOR MODEL}

Our analysis uses an HTS conductor based on a most basic design which is the starting point of a simulation model and not necessarily a realistic conductor design.

The cable consists of a stack of 1233 (RE)BCO tapes at the following operation conditions: magnetic field $B_{o p}=10 \mathrm{~T}$, temperature $T_{o p}=50 \mathrm{~K}$, operating current $I_{o p}=16.5 \mathrm{kA}$ ( $=50 \%$ of the cable critical current). The current sharing temperature is $T_{C S}=58 \mathrm{~K}$. The conductor includes a cooling channel in contact with the cable, with forced flow of gaseous helium.

The reference tape, which we have taken as an example to test our method, is the SuperPower $2 G$ (RE)BCO-HTS Tape $S C S 4050$. This consists of layers of (RE)BCO $(1 \mu \mathrm{m})$, copper stabilizer $(40 \mu \mathrm{m})$, Hastelloy C-276 substrate $(50 \mu \mathrm{m})$, silver overlayer $(2 \mu \mathrm{m})$, and buffer stack $(\approx 0.2 \mu \mathrm{m})$. The tape width is $4 \mathrm{~mm}$. With the tape data provided by the manufacturer's web site we have established a scaling relation for the critical current density as a function of magnetic field and temperature taking as a reference value the critical current of $100 \mathrm{~A}$ ( $77 \mathrm{~K}$, self-field). Mechanical and physical properties of (RE)BCO and Hastelloy [4] have been included in the CryoSoft package.

The operating conditions are based on general cryogenic considerations (e.g., considerably lower cryogenic costs are achieved at temperatures $\geq 50 \mathrm{~K}$ when using coated conductors for fusion devices) and on the tape critical current density rather than on a detailed thermodynamic analysis. These conditions are similar to those used for the development of a coated conductor cable by the Roebel technique at the Karlsruhe Institute of Technology [5], and can be regarded as the starting point for a Torodial Field magnet design of the next fusion device, DEMO.

\section{Simulation Models}

The quench behavior of the HTS conductor is investigated using two simulation models with simplifying assumptions as a compromise between reliability and cost efficiency:

- Detailed model (DM) consisting of 5 parallel tapes, of which 3 have the physical dimensions and characteristics of the reference tape, while the other 2 simulate the rest of the cable (megatapes), as shown in Fig. 1. This model is used to study at $I=I_{o p}$ the transverse (tape to tape) and longitudinal (along the conductor length) quench propagation, with and without the effect of helium forced flow, on a short conductor length of $2 \mathrm{~m}$.

- Homogeneous model (HM) consisting of one megatape including all 1233 tapes in perfect thermal and electrical contact. This model is used to study along the full conductor 


\begin{tabular}{|c|c|c|c|c|}
\hline $\begin{array}{l}\text { Megatape } \\
\text { (615 tapes) }\end{array}$ & T9/E5 & T10 & \multirow{5}{*}{$\mathrm{H} 1$} & $\begin{array}{l}\text { Materials in } \\
\text { Thermal components }\end{array}$ \\
\hline \multirow{3}{*}{$\begin{array}{l}\text { Single tape } \\
\text { Single tape } \\
\text { Single tape }\end{array}$} & T7/E4 & T8 & & \multirow{2}{*}{$\begin{array}{c}\text { T1, T3,T5,T7,T9: } \\
\text { (RE)BCO + } \\
\text { Copper }\end{array}$} \\
\hline & T5/E3 & T6 & & \\
\hline & T3/E2 & T4 & & $\mathrm{T} 2, \mathrm{~T} 4, \mathrm{~T} 6, \mathrm{~T} 8, \mathrm{~T} 10:$ \\
\hline $\begin{array}{l}\text { Megatape } \\
\text { (615 tapes) }\end{array}$ & T1/E1 & T2 & & $\begin{array}{l}\mathrm{H} 1: \mathrm{He} \\
\text { NOT TO SCALE }\end{array}$ \\
\hline
\end{tabular}

Fig. 1. Cross section of the DM with ten thermal (T1-T10), five electric (E1-E5), and one hydraulic (H1) components. The model HM, not shown here, is composed of two thermal components (T1-T2, each of 1233 tapes) and $\mathrm{H} 1$, without electric components.

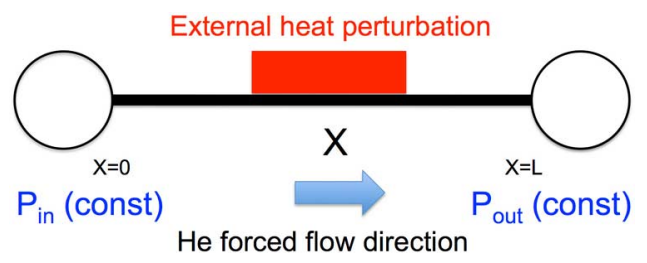

Fig. 2. HM and DM models in the longitudinal direction along the conductor length. Details of the external heat perturbation are given in Table II.

length of $300 \mathrm{~m}$ (a) the peak quench characteristics, including detection of the normal zone and current dump, and (b) the steady state hydraulic conditions.

In both models the tape is modeled in THEA by 2 thermal components: (a) (RE)BCO and copper and (b) Hastelloy. The conductor is modeled in 1-D along the coordinate $X$, i.e. the distance from the helium inlet (Fig. 2). The simulation ends at time $t_{\text {end }}(15 \mathrm{~s}$ in the DM, $90 \mathrm{~s}$ in the HM).

The electrical field of the power fit to the voltage-current curve for the critical current transition is $E_{0}=100 \mu \mathrm{V} / \mathrm{m}$. The copper residual resistivity ratio is $R R R=100$. The DittusBolter correlation is used for the heat transfer coefficient model. The cryogenic system consists of reservoirs at constant pressure, located at the He inlet and outlet. The inlet pressure is $10 \mathrm{bar}$ and the pressure drop $1 \mathrm{bar}$ for $100 \mathrm{~m}$ of length. Other assumptions are: $\mathrm{AC}$ losses and strain effect are neglected, the magnetic field is constant along $X$, structural and insulation components are not included.

The perturbation is deposited into the central tape (T5 in DM, T1 in HM) along $X$ for a length $L_{P}$ (Table II), begins at time $t=$ 0 and is constant in time. The power to trigger the quench is $Q=$ $1000 \mathrm{~W} / \mathrm{m}$ in scenario $\mathrm{H}$ and $100 \mathrm{~W} / \mathrm{m}$ in all other scenarios; these values are not an accurate estimate of the minimum quench energy since a stability analysis is beyond the scope of this work.

Each thermal component is assumed to be in thermal contact only with the adjacent ones through two thermal resistances, i.e. between $(\mathrm{RE}) \mathrm{BCO} / \mathrm{Cu}$ and Hastelloy $\left(T R_{S H}\right)$ and between tape and tape $\left(T R_{T T}\right)$ (Fig. 3). Based on a simple equivalent heat conduction model and data in the literature [6], we take the former in the range of $10^{-4}$ to $10^{-2} \mathrm{Km} / \mathrm{W}$ and the latter in the range 0.25 to $250 \mathrm{Km} / \mathrm{W}$. These values are approximate estimates which will need to be confirmed experimentally.

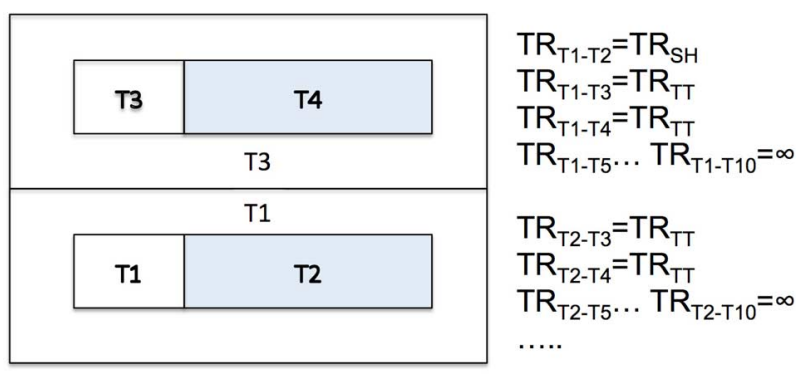

Fig. 3. Model of thermal resistances between thermal components. Only the first two tapes of the DM are shown for simplicity. Fig. 1 lists the materials in the thermal components.

TABLE I

RELEVANT MODEL AND SIMULATION DATA

\begin{tabular}{llcc}
\hline \hline Parameter & Unit & HM & DM \\
\hline Conductor length & $\mathrm{m}$ & 300.0 & 2.0 \\
Cu cross section (single tape) & $\mathrm{mm}^{2}$ & - & 0.1717 \\
Cu cross section (megatape) & $\mathrm{mm}^{2}$ & 211.7 & 105.6 \\
(RE)BCO cross sec. (single tape) & $\mathrm{mm}^{2}$ & - & 0.004 \\
(RE)BCO cross sec. (megatape) & $\mathrm{mm}^{2}$ & 4.932 & 2.46 \\
Hastelloy cross sec. (single tape) & $\mathrm{mm}^{2}$ & - & 0.2 \\
Hastelloy cross sec. (megatape) & $\mathrm{mm}^{2}$ & 246.6 & 123.0 \\
Helium cross section & $\mathrm{mm}^{2}$ & 114.7 & 114.7 \\
Hydraulic diameter & $\mathrm{mm}^{2}$ & 1.983 & 1.983 \\
Ref. Thermal resistance $T R_{S H}$ & $\mathrm{Km}_{\mathrm{WW}}$ & $2 \times 10^{-3}$ & $2 \times 10^{-3}$ \\
Ref. Thermal resistance $T R_{T T}$ & $\mathrm{Km} / \mathrm{W}$ & 0 & 8.0 \\
\hline THEA thermal components & - & 2 & 10 \\
THEA electric components & - & - & 5 \\
THEA hydraulic components & - & 1 & 1 \\
THEA elements & - & 1000 & 600 \\
\hline \hline
\end{tabular}

\section{RESULTS AND DisCUSSION}

\section{A. Reference Case (Detailed Model)}

The input data of the "Reference Case" of the DM model and its results are shown in Table I and Fig. 4, respectively.

As heat is deposited, the temperature in T5 (see Fig. 1) goes up, the critical current density goes down, the current is redistributed and the normal zone propagates (a) among tapes in the same cross section (transverse propagation) and (b) along the conductor length (longitudinal propagation). The temperature gradient among tapes in the heated zone is an indicator of the transverse propagation, and the normal zone length along $X(N Z L)$ of the longitudinal propagation.

The transverse propagation from $\mathrm{T} 5$ into the adjacent tapes (T3, T7) is almost instantaneous. In the same conductor cross section it takes $\approx 6.8$ seconds for the perturbation to begin its expansion into the megatapes (T1, T9). The longitudinal propagation does not start at the same time in all tapes, i.e. at $\approx 5 \mathrm{~s}$ in $\mathrm{T} 3, \mathrm{~T} 5, \mathrm{~T} 7$ and $\approx 6.8 \mathrm{~s}$ in $\mathrm{T} 1, \mathrm{~T} 9$ as shown by the propagation velocity $N Z V=d(N Z L) / d t$ in Fig. 4.

The peak temperature is $\approx 300 \mathrm{~K}$ in T5 at $X=1.1 \mathrm{~m}(\approx 80 \mathrm{~K}$ in T3-T7), a value above $200 \mathrm{~K}$ which is considered a limit to avoid critical thermal stresses. After the quench is fully developed the $N Z V \approx 10 \mathrm{~cm} / \mathrm{s}$ and constant in time. The temperatures in Hastelloy and in (RE)BCO/Cu are practically the same because of the good thermal contact between these two components. 

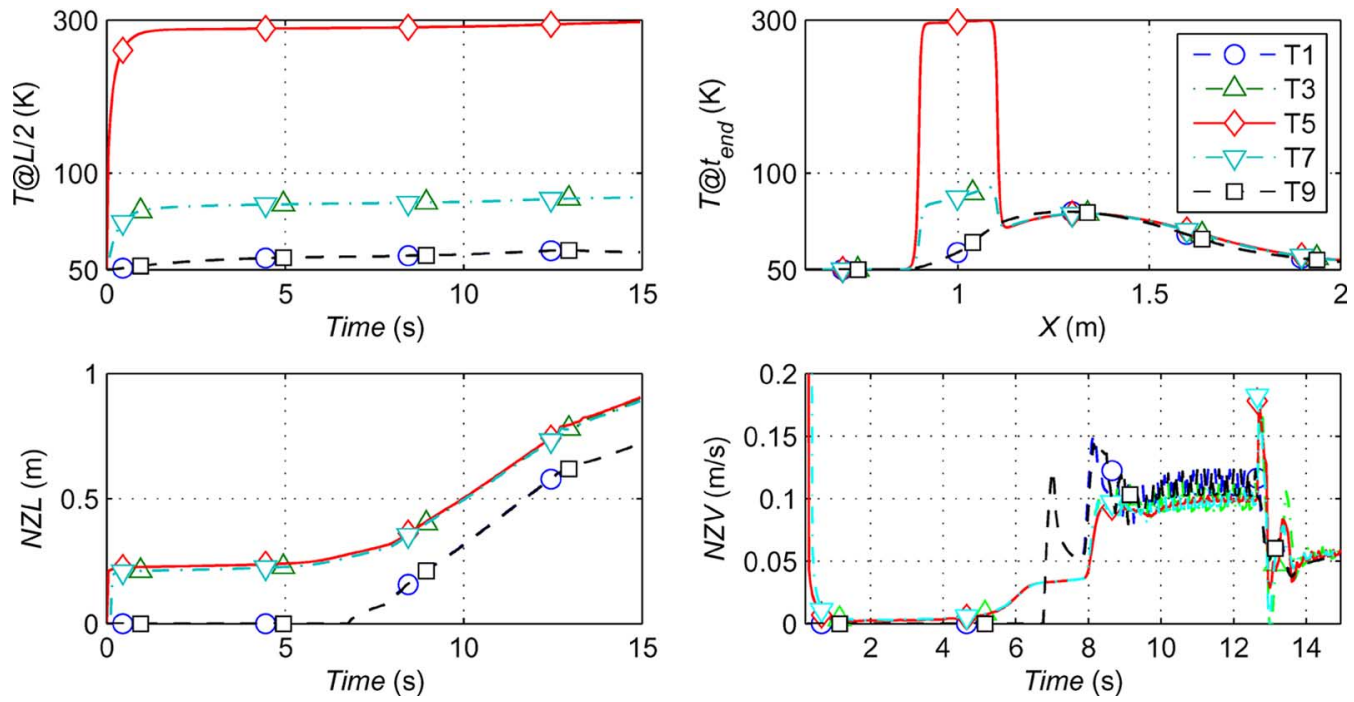

Fig. 4. Reference case. Top: (Left) Time history of the conductor temperature at $X=L / 2$ and (right) distribution along the conductor length of the conductor temperature at $t_{\text {end }}$. Bottom: Time history (left) of the normal zone length and (right) of the normal zone propagation velocity. The oscillations of $N Z V$ ( $10 \mathrm{~s}<$ $t<13 \mathrm{~s}$ ) are due to numerical derivation of $N Z L$. Only the thermal components with (RE)BCO/Cu are shown, i.e. T1, T3, T5, T7, and T9 in the legend. The legend is the same for all plots.

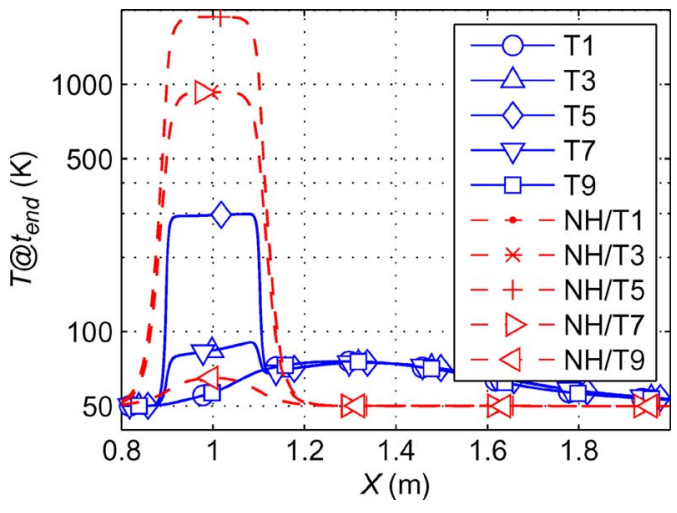

Fig. 5. Comparison of the reference case with the case with no helium (NH). Distribution along the conductor length of the temperature of $(\mathrm{RE}) \mathrm{BCO} / \mathrm{Cu}$ (thermal components $\mathrm{T} 1, \mathrm{~T} 3, \mathrm{~T} 5, \mathrm{~T} 7$, and T9) at $t_{\text {end }}$. The extremely high temperatures of the $\mathrm{NH}$ model are an artifact.

The consistency of the electrical model is checked by the difference between $I_{o p}$ and the sum of currents in the electrical components which is $\approx 0$. The total voltage is $0.10 \mathrm{mV}$ at $t=8 \mathrm{~s}$ and $10 \mathrm{mV}$ at $t=10 \mathrm{~s}$.

\section{B. Effect of Helium Flow (Detailed Model)}

If there is no cooling (DM model without H1) the normal zone is practically confined to its initial position, with an extremely low longitudinal propagation $\left(N Z L=L_{P}\right.$ and $N Z V \approx 0$ ). The comparison with the Reference Case clearly shows that the He flow is beneficial for moving the normal zone downstream, thus reducing the peak temperature in the heated zone (Fig. 5).

These results are not substantially different if the model is analysed with (a) no coolant or (b) the coolant at rest. This is due to the low heat capacity of the helium compared to the high heat capacity of (RE)BCO and copper at temperatures $>50 \mathrm{~K}$.

In our model helium is in contact with all tapes, a condition which could be achieved for example by an HTS cable-in-conduit conductor. The cooling system could therefore be opti-

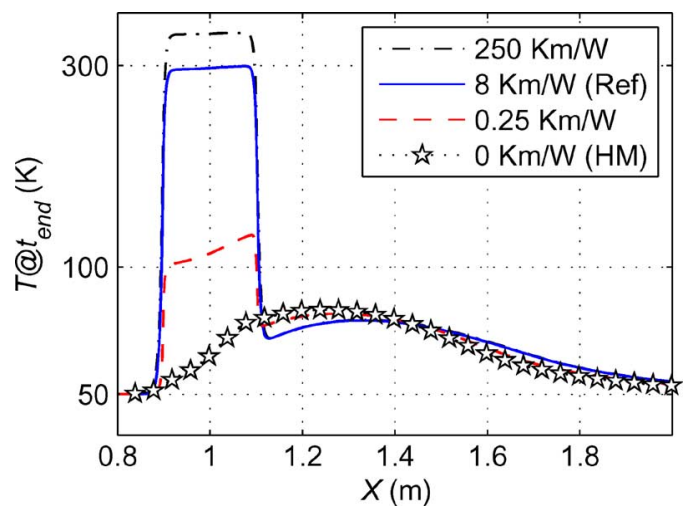

Fig. 6. Effect of the thermal resistance between tapes $T R_{T T}$. Distribution along the conductor length of the temperature at $t=t_{e n d}$ in the heated tape, i.e. T5 in the DM and T1 in the HM model (special case: scenario H, no current dump, 4000 THEA elements; see below).

mized to enhance the "quench forward" process and facilitate the detection of the presence of a non-recovering resistive zone.

\section{Parametric Study (Detailed Model)}

The transverse quench propagation is sensitive to variation of the thermal resistance between tapes $T R_{T T}$, i.e. the peak temperature in the heated zone is considerably reduced (i.e. from $320 \mathrm{~K}$ to $120 \mathrm{~K}$ ) as the thermal contacts between tapes is reduced from 250 to $0.25 \mathrm{~K} \mathrm{~m} / \mathrm{W}$ (Fig. 6). The results of $N Z L$ and $N Z V$ are almost unaffected by a variation of this parameter.

The sensitivity of the quench propagation results to variation of the thermal resistances between (RE)BCO/Cu and Hastelloy is very limited $(<5 \%)$ in the evaluated range of $T R_{S H}$. The same sensitivity is completely negligible to variation of the electrical conductance in the range $10^{6}$ (Reference Case) to $10^{3}(\Omega \mathrm{m})^{-1}$.

During a quench the resistive component of the total voltage dominates over the inductive component; therefore the effect 
TABLE II

PERTURBATION SCENARIOS

\begin{tabular}{cccc}
\hline \hline Scenario & $\begin{array}{c}\text { Centered } \\
\text { At Location }\end{array}$ & $\begin{array}{c}\text { Model HM } \\
\text { Length } L_{P}(\mathrm{~m})\end{array}$ & $\begin{array}{c}\text { Model DM } \\
\text { Length } L_{P}(\mathrm{~m})\end{array}$ \\
\hline $\mathrm{A}$ & - & 300 & - \\
$\mathrm{B}$ & $X=L / 2$ & 150 & - \\
$\mathrm{C}$ & $X=L / 2$ & 10 & - \\
$\mathrm{D}$ & $X=0$ & 10 & - \\
$\mathrm{E}$ & $X=L$ & 10 & - \\
$\mathrm{F}$ & $X=L / 2$ & 2 & - \\
\hline $\mathrm{H}$ & $X=L / 2$ & 0.2 & 0.2 \\
\hline \hline
\end{tabular}

of the inductive element distribution is only responsible for a second order effects.

\section{Homogeneous Model}

The steady state hydraulic conditions are: the helium mass flow rate $\approx 3.7 \mathrm{~g} / \mathrm{s}$ and its residence time in the $300 \mathrm{~m}$ long conductor $\approx 2$ minutes.

A simplified detection model is used to assess the peak quench characteristics. Until detection of the normal zone at time $t_{d e t}$ the current is $I=I_{o p}$; at this point in time the current dump begins according to

$$
I=I_{o p} e^{-t / \tau_{d u m p}}
$$

with the time constant $\tau_{\text {dump }}$ in the range 10 to $5 \mathrm{~s}$. At this stage of the analysis we vary the input parameter $t_{\text {det }}$ and calculate $a$ posteriori the voltage at detection $V_{d e t}$. In a more detailed analysis we will use a voltage criterion to trigger the current dump, as done for the EDIPO superconducting magnet system [7].

In the perturbation scenario $\mathrm{A}\left(L_{P}=L\right)$ the peak He pressure is $<11$ bar (approximately the steady state value) and the peak mass flow rate is $6.5 \mathrm{~g} / \mathrm{s}(\approx 2$ times the steady state value) with no flow reversal. These values are extremely low although the simulation of all scenarios of Table II confirms that the scenario $\mathrm{A}$ is the most severe (and not likely to occur in reality) as far as these parameters are concerned. The fundamental difference between forced flow with gaseous helium and with supercritical helium, as in LTS cable-in-conduit conductors, is that in the latter the pressure and mass flow rate during a quench can reach extremely high values because of the large change of density whereas in the former there is no such change.

\section{SUMMARY}

The quench analysis of a high current HTS conductor model, which could be regarded as the starting point for a fusion conductor, was performed using two simulation models with the CryoSoft code THEA. This analysis shows:

i) The helium flow has a beneficial impact on the longitudinal expansion of the normal zone and the resulting reduction of the peak temperature in the heated zone. Therefore there is a margin to optimize this effect, e.g. by selection of the channel size and mass flow rate.

ii) The thermal resistance between tapes has an impact on the transverse propagation of the normal zone within the conductor cross section, e.g. the peak temperature is uncritical $(<200 \mathrm{~K})$ for resistances $<1 \mathrm{Km} / \mathrm{W}$.

iii) The peak helium quench pressure and mass flow rate are not critical because there is no large change of density in the gaseous helium.

Based on these promising results, we plan to enhance the simulation model to investigate more realistic conductor configurations.

\section{ACKNOWLEDGMENT}

The authors are grateful to Ciro Calzolaio and Stephen March (CRPP) for stimulating discussions and to Valery Markushin (PSI) for the IT support.

\section{REFERENCES}

[1] X. Wang, A. Caruso, and M. Breschi et al., "Normal zone initiation and propagation in Y-Ba-Cu-O coated conductors with cu stabilizer," IEEE Trans. Appl. Supercond., vol. 15, no. 2, pp. 2586-2589, 2005.

[2] M. Marchevsky, Y.-Y. Xie, and V. Selvamanickam, "Quench detection method for 2G HTS wire," IOP Supercond. Sci. Technol., vol. 23, no. 034016, 2010

[3] L. Bottura, C. Rosso, and M. Breschi, "A general model for thermal, hydraulic, and electric analysis of superconducting cables," Cryogenics, vol. 40, no. 8-10, pp. 617-626, 2000.

[4] J. Lu, E. S. Choi, and H. D. Zhou, "Physical properties of Hastelloy C-276 at cryogenic temperatures," Journal of Applied Physics, vol. 103, no. $064908,2008$.

[5] S. I. Schlachter, W. Goldacker, F. Grilli, R. Heller, and A. Kudymow, "Coated conductor Rutherford cables (CCRC) for high-current applications: Concept and properties," in This Conference (2MP2B-07), 2010.

[6] E. Gmelin, M. Asen-Palmer, M. Reuther, and R. Villar, "Thermal boundary resistance of mechanical contacts between solids at sub-ambient temperatures," J. Phys. D: Appl. Phys., vol. 32, pp. R19-R43, 1999.

[7] C. Marinucci, M. Calvi, L. Bottura, P. Bruzzone, and R. Herzog, "Quench analysis of the EDIPO high field superconducting dipole magnet," IEEE Trans. Appl. Supercond., vol. 18, no. 2, pp. 200-203, 2008. 